\title{
Expresión facial y reconocimiento de emociones en lactantes
}

\author{
JAIME IGLESIAS \\ ANGELA LOECHES \\ JUAN SERRANO \\ Universidad de Madrid
}

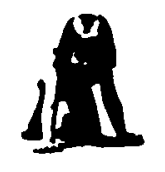

Resumen

En esta revisión presentamos los resultados e implicaciones de los trabajos realizados por nuestro equipo de investigación sobre expresión y reconocimiento de emociones en la infancia. Dichos trabajos se enfocan desde una perspectiva psicobiológica, asumiéndose que un conjunto de emociones consideradas básicas son estados discretos del organismo, determinados genéticamente y regulados por estructuras nerviosas subcorticales, que tienen un valor adaptativo para los individuos bajo determinadas circunstancias estimulares. Situándonos dentro de la tradición de estudios que apoyan la hipótesis de la universalidad de la expresión facial, aportamos una serie de datos que nos conducen a concluir que los lactantes son capaces de expresar $y$ de discriminar desde los tres meses de edad las emociones de alegria, ira, miedo, sorpresa, desagrado y tristeza. Estos datos ban sido obtenidos utilizando un paradigma de habituación visual y un método objetivo de análisis denominado Facial Action Coding System. A pesar de contar con estas pruebas empiricas, terminamos matizando que aún desconocemos los factores evolutivos que originan las expresiones emocionales.

Palabras clave: Emoción, Expresión facial, Comunicación no verbal, Desarrollo emocional, Conducta infantil.

\section{Facial expression and emotional recognition in infants}

\section{Abstract}

In this paper we present the research developed by our group on facial expression and recognition of emotion in infancy. Based upon a psychobiological framework, we assume that the so named «basic emotions» are discrete organismic states phylogenetically shaped and subcortically mediated, that facilitate the adaptation of individuals under specific stimulating conditions. Reviewing the empirical evidence that sup. ports the hypothesis of the universality of facial expression, our data lead us to conclude that infants at the early age of three months are able to express and recognize the emotions of bappiness, anger, fear, surprise, disgust and sadness. These data have been obtained by means of methods of objective measurement as the Facial Action Coding System, and the visual babituation-recovery paradigm. However despite these empirical data, we finish by pointing out that the evolutionary mechanisms that originate emotional expression remain unknown.

Keywords: Emotion, Facial expression, Nonverbal communication, Emotion development, Infant behavior.

Agradecimientos: Los estudios experimentales realizados por nuestro equipo de investigación se han llevado a cabo gracias a la financiación recibida de la Dirección General de Investigación Cien. tífica y Técnica (DGIC y T P886-0116) y de la Caja de Madrid. Partes complementarias de este trabajo se presentaron en el II Congreso Nacional de Etologia, Sevilla, 27-30 septiembre de 1988.

Dirección del Autor: Universidad Autónoma de Madrid. Facultad de Psicología. Dpto. Psicología Biológica y de la Salud. Cantoblanco. 28049 Madrid.

Original recibido: mayo 1989. Revisión recibida: agosto 1989. Aceptado: septiembre 1989. 


\section{INTRODUCCION}

El comportamiento emocional recibe en el momento actual una gran atención por parte de las Neurociencias (Smith y DeVito, 1984), de la Sociología y la Antropología (Lutz y White, 1986; Scheff, 1983) y, sobre todo,de la Psicología (Blanchard y Blanchard, 1988; Leventhal y Tomarken, 1986). En este último caso es especialmente notable el número de trabajos experimentales, textos y monografías publicados en los últimos años (Kleinginna y Kleinginna, 1981; Leventhal y Tomarken, 1986), además de la creación reciente de dos revistas especializadas, denominadas Motivation and Emotion, editada por Plenum Press, y Cognition and Emotion, publicada por Lawrence Erlbaum Associates. Esta eclosión se debe principalmente al surgimiento de nuevas técnicas que permiten analizar objetivamente las emociones (Izard, 1982; Levi, 1975; Plutchik, 1980; Scherer y Ekman, 1982), paralelamente al desarrollo de diversas teorías psicológicas sobre la naturaleza y función de los procesos emocionales (Plutchik y Kellerman, 1980; Scherer y Ekman, 1984).

En este contexto, destaca en especial el interés mostrado por los psicobiólogos por describir las pautas estereotipadas de conducta emocional de las diversas especies en condiciones naturales y experimentales, así como también por determinar los mecanismos neurofisiológicos subyacentes, su evolución y significado adaptativo (Blanchard y Blanchard, 1988). También sobresale la preocupación creciente de los psicólogos evolutivos y cognitivos por descubrir las relaciones existentes entre los procesos cognitivos y emocionales y determinar su desarrollo (Clark y Fiske, 1982), el interés actual de los psicólogos sociales por analizar la influencia del contexto en la regulación de la expresión emocional (Fernández-Dols, Iglesias y Mallo, en prensa; Frijda, 1986; Shaver, 1984) y por último, el de los psicólogos de la personalidad y clínicos por dilucidar los componentes emocionales de los rasgos de personalidad y de las diferentes psicopatologías (Blankstein, Pliner y Polivy, 1980; Weiner, 1986). De todo ello se desprende el carácter interdisciplinario del campo de la emoción, dentro del cual se ha impuesto en los últimos años la perspectiva teórica propia de la Psicobiologia, cuyos presupuestos básicos pasamos a detallar a continuación, ya que en ellos se fundamenta la hipótesis de la universalidad de la expresión facial que da lugar a nuestras investigaciones con lactantes.

\section{BASES CONCEPTUALES DEL ESTUDIO PSICOBIOLOGICO DE LAS EMOCIONES}

La Psicobiología, situada en un punto intermedio entre la Psicología y las Neurociencias, se caracteriza por su independencia académica en relación con las distintas escuelas psicológicas y por definir las emociones dentro de un marco de pensamiento neodarwiniano, lo que significa poner un especial énfasis en su naturaleza filogenética y en su carácter adaptativo para afrontar determinadas demandas ambientales (Fox y Davidson, 1984; Domura, 1986; Panksepp, 1982). En particular, se supone que ciertas emociones consideradas básicas (alegría, ira, miedo, tristeza y para algunos autores desagrado y sorpresa) se han desarrollado en el curso de la evolución con el objeto de favorecer la comunicación intraespecífica y la supervivencia de los individuos, especialmente durante las primeras interacciones madre-hijo (Trevarthen, 1985).

De acuerdo con lo que acaba de señalarse, los postulados básicos sobre los 
que descansan los estudios psicobiológicos de las emociones han sido puestos de relieve recientemente por Panksepp (1982), y pueden resumirse en los tres siguientes. Primero, se supone que las emóciones básicas resultan de la actividad de determinados circuitos neurales del hipotálamo y el sistema límbico, desarrollados en las primeras etapas de la evolución del cerebro de los mamíferos para responder de forma incondicionada ante estímulos de especial relevancia para la supervivencia de los individuos. Segundo, se asume que tales circuitos neurales organizan y guían la conducta mediante la activación e inhibición de patrones específicos de respuestas, incluyendo cambios subjetivos, somáticos y vegetativos, todos los cuales cumplen una función determinada ante la presencia de dichos estímulos. Tercero, se supone también que los circuitos neurales propios de cada emoción mantienen interacciones recíprocas con los mecanismos cerebrales que subyacen a los procesos cognitivos superiores, de forma que las respuestas emocionales innatas se pueden condicionar en el curso del desarrollo a circunstancias estimulares emocionalmente neutrales por medio del refuerzo.

En consecuencia, desde una perspectiva psicobiológica se defiende que las emociones son parte de la conducta motivada, equiparables en cierto modo con el aprendizaje en lo que respecta a su papel de guía del comportamiento adaptativo, no estando necesariamente supeditadas a los procesos cognitivos superiores (Öhman, 1987; Plutchik, 1980). Lo más alentador de esta perspectiva es que, asumiendo la continuidad evolutiva de los mecanismos cerebrales que elaboran las emociones podemos establecer comparaciones entre adultos y niños, sujetos normales y deficientes físicos o psíquicos, así como también entre el hombre y otros organismos animales, con el objeto de analizar cualquier aspecto de la conducta emocional.

Como se ha indicado anteriormente, las pruebas experimentales disponibles, aportadas por la Psicología Fisiológica y la Neuropsicología, nos permiten afirmar que tales mecanismos cerebrales radican en núcleos neuronales subcorticales, pertenecientes al hipotálamo y al cerebro límbico. Así, se ha observado que las conductas instintivas de naturaleza emocional permanecen inalteradas en animales decorticados poco después del nacimiento y que, con alguna excepción, las lesiones del tejido subcortical en humanos producen cambios similares a los que resultan de su manipulación experimental en otros mamíferos (Isaacson, 1982; Kupfermann, 1985). En conclusión, parece que, desde un punto de vista funcional, las estructuras subcorticales son similares en los mamíferos y constituyen la base neurobiológica de los cambios que se observan en los distintos sistemas de respuesta de los organismos durante los procesos emocionales.

El mejor ejemplo de lo que acaba de indicarse lo constituyen las expresiones . faciales de las emociones denominadas básicas, las cuales son respuestas muy estereotipadas y con un claro valor comunicativo en los primates humanos y no humanos, que se encuentran reguladas originalmente por neuronas subcorticales pertenecientes al sistema motor extrapiramidal (Rinn, 1984). A pesar de la existencia de diversas pruebas en favor de su universalidad, algunos autores mantienen aún la idea de que tales expresiones emocionales son señales convencionales y variables de una cultura a otra, que no están ligadas directamente a la conducta emocional y que se encuentran determinadas por procesos cognitivos de origen cortical (véase, p. e., Valle-Inclán, 1983). Si bien los datos actualmente disponibles en favor de la universalidad de la expresión facial no implican necesariamente su innatismo, como se verá en un apartado posterior, es posible que estos autores estén confundiendo el problema de la expresión emocional es- 
pontánea con el de la expresión deliberada o voluntaria (Iglesias, Naranjo, Picazo y Ortega, 1986).

\section{DESARROLLO HISTÓRICO DE LAS INVESTIGACIONES SOBRE EXPRESION FACIAL Y EMOCION}

Las investigaciones sobre expresión facial y emoción tienen su origen en Charles Darwin (1872/1984; 1877/1983), Darwin compara el comportamiento emocional de los animales y el hombre, destaca la existencia de elementos comunes en la expresión emocional de sujetos humanos pertenecientes a distintas culturas, hace hincapié en la similitud del repertorio expresivo de niños ciegos $\mathrm{y}$ videntes $\mathrm{y}$, finalmente, es el primero que observa de forma sistemática la aparición temprana de los gestos emocionales en el niño de corta edad. En conclusión, Darwin mantiene que las expresiones faciales de las emociones son universales y que han sido seleccionadas en el curso de la evolución para transmitir mensajes discretos acerca del estado interno de su emisor, resultando por ello claves en la regulación de la comunicación intraespecífica y la supervivencia de los individuos.

Medio siglo después de la publicación de los escritos darwinianos, la hipótesis de la universalidad de la expresión facial se incorpora a las polémicas abiertas acerca de la determinación genética o ambiental del comportamiento humano. Así, el interés por las investigaciones sobre la expresión facial de emociones se encuentra ya en psicólogos como Allport (1924/1967), Goodenough (1931), Landis (1929) Sherman (1927), y Woodworth (1938), entre otros, sin que entonces pudieran aportarse datos concluyentes acerca de las relaciones entre expresión facial y emoción, la universalidad de las expresiones faciales y su posible carácter innato. Sin embargo, durante aquella época prevalecieron las opiniones de Landis (1929), y Sherman (1927), a pesar de estar basadas en trabajos muy deficientes desde un punto de vista metodológico. Estos autores, tras observar que los juicios sobre expresiones faciales de emociones tan sólo eran acertados cuando se conocía el estímulo presentado en cada caso, concluyeron que la expresión facial no era un indicativo suficiente para juzgar la presencia de una emoción. El tema perdió interés en los años siguientes.

Con posterioridad, Hebb (1946) defendió que las expresiones faciales de las emociones podian ser identificadas sin percibir su causa, en función de la familiaridad de los observadores con el individuo que la expresa y el contexto social en que ocurren. Estas afirmaciones aumentaron el escepticismo de los psicólogos por los estudios sobre la expresión de las emociones. En este estado de opinión, las investigaciones fueron muy escasas, exceptuando los trabajos de Schloesberg (1954), quien clasificó las distintas categorías emocionales según tres dimensiones bipolares, a saber, el nivel de activación, el tono hedónico y la disposición atencional.

El interés por la expresión facial no renace hasta que Tomkins $(1962,1963)$ da a conocer su teoría de las emociones discretas (una versión actualizada puede verse en Tomkins, 1982). Según esta teoría, las emociones tienen un origen subcortical y se encuentran definidas por patrones específicos de cambios faciales, pudiendo ser identificadas con precisión por cualquier individuo a partir de su mera presencia y en ausencia de otros indicios, siempre que representen fiablemente afectos innatos. Dicha identificación por parte del receptor se supone 
que no tiene lugar en función del estímulo o del contexto social en que ocurren, ni de la familiarización previa con su emisor, sino que se basa en el mensaje que transmite la expresión emocional acerca de la conducta futura de este último. Tal anticipación no implica necesariamente experimentar el sentimiento de la otra persona, sino tan sólo la formación de expectativas acerca de sus tendencias de aproximación, enfrentamiento o escape.

Tomkins influyó directamente sobre Ekman e Izard, motivándoles a la realización de investigaciones transculturales y a continuar, en definitiva, la línea ya trazada por Darwin en el estudio de la expresión emocional. Ambos autores, por separado, comprobaron la existencia en diversas culturas de al menos seis expresiones faciales universales, correspondientes a seis de las emociones que tanto Darwin como Tomkins había considerado innatas: alegría, ira, miedo, sorpresa, desagrado y tristeza (Ekman y Friesen, 1971; Izard, 1971). Poco después, Ekman (1973) edita Darwin and facial expression, donde distintos autores revisan las investigaciones postdarwinianas sobre expresión facial en primates no-humanos, expresión emocional en niños ciegos y videntes, y también los estudios sobre expresión facial y emoción en sujetos humanos pertenecientes a distintas culturas, contrastándolos directamente con las observaciones pioneras de Darwin.

Los avances registrados en este campo de investigación son recibidos con especial interés por los llamados «etólogos humanos», uno de cuyos propósitos fundamentales es la explicación del comportamiento social humano según hipótesis derivadas de la teoría de la evolución. Estos autores valoran especialmente los resultados de los estudios transculturales e insisten en que las expresiones faciales de las emociones son conductas adquiridas a lo largo de la evolución por su valor adaptativo. Eibl-Eibesfeldt $(1972,1975)$, continúa realizando investigaciones transculturales y estudios sobre la expresión emocional de niños sordomudos ciegos y de otros con diversos déficits sensoriales, concluyendo que el aprendizaje visual no es una condición necesaria para el desarrollo de la expresión emocional.

Esta línea de trabajo no es seguida por otros etólogos. Mientras que algunos restringen su campo de trabajo al estudio de la expresión facial y la comunicación visual en primates no humanos (Andrew, 1972; Redican, 1975; Van Hooff, 1972) otros se limitan a desarrollar catálogos descriptivos de expresiones faciales mostradas por niños de edades escolares en diversas situaciones naturales (Blurton-Jones, 1971; McGrew, 1972). De este modo, los estudios empíricos sobre el estudio de la expresión facial y la emoción se desarrollan en ausencia de un marco teórico àdecuado, que pueda agrupar a los autores interesados en este campo y potenciar la realización de investigaciones convergentes.

Son precisamente Ekman (1977), Izard (1977) y Plutchik (1980) quienes, más tarde, proponen o amplían teorías previas de la emoción en consonancia con los presupuestos originales de Darwin. Estos autores defienden la existencia de un número reducido de seis a ocho emociones básicas, determinadas genéticamente, de cuya combinación surgen las denominadas emociones sociales (en la Figura 1 pueden verse las emociones básicas propuestas por Plutchik, 1980). Dichos autores consideran además que la cara constituye el lugar principal donde se asientan las emociones y defienden que el valor adaptativo de las expresiones faciales radica en que transmiten fiablemente el estado emocional de su emisor y su tendencia conductual, interviniendo directamente en el establecimiento y regulación de los contactos sociales. Así, mientras que la expresión de ira conlleva una intención de ataque o confrontación en relación con el evento 
FIGURA 1

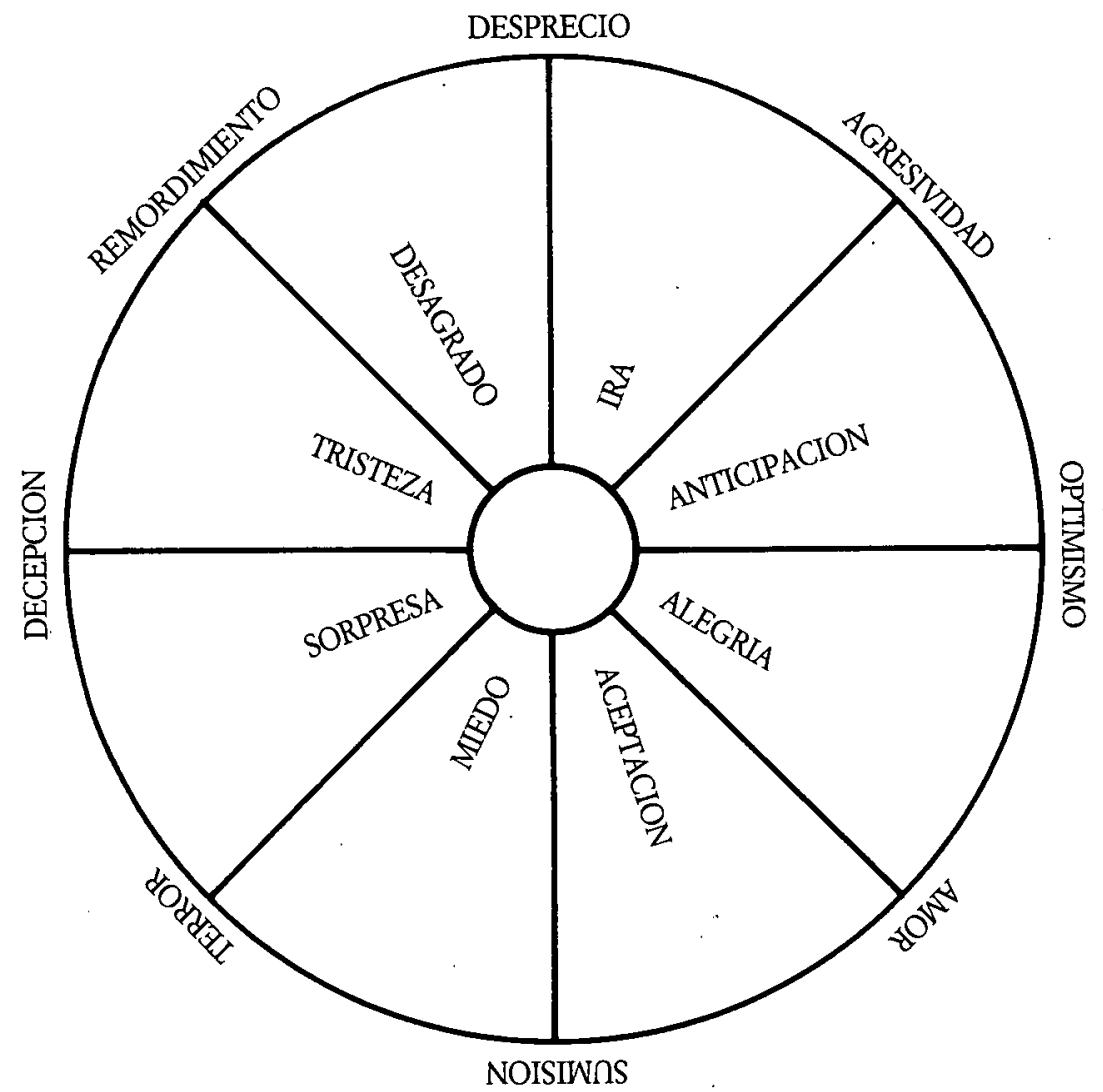

Representación circular de las emociones según Plutchik (1980), atendiendo a su similaridad y antagonismo, con indicación de las posibles emociones derivadas a partir de la combinación de pares adyacentes de emociones básicas.

frustrante, la expresión de miedo transmite la necesidad de escapar o recibir protección ante alguna amenaza; a diferencia de estas dos expresiones, la alegría refleja una intención afiliativa en relación, generalmente, con un estímulo social familiar (Izard, 1977).

Dado el papel que sobre todo Ekman e Izard atribuyen a la expresión facial en sus respectivas teorías, estos autores se esfuerzan por elaborar técnicas objetivas de codificación de acciones faciales, teniendo en cuenta la anatomía funcional de la cara. La publicación reciente de dos códigos, el FACS de Ekman y Friesen (1978), y el MAX de Izard (1979), que permiten describir las acciones visibles de los músculos faciales, han atraído el interés de los investigadores por el estudio empírico de las relaciones entre expresión facial y emoción y, en particular, sobre la supuesta universalidad y el innatismo de la expresión emocional.

\section{SITUACION ACTUAL DE LA HIPOTESIS DE LA UNIVERSIDAD DE LA EXPRESION FACIAL}

La polémica acerca de si las expresiones faciales son universales o específicas de cada cultura se encuentra actualmente resuelta para la mayoría de los in- 
vestigadores. Observaciones en uno $u$ otro sentido son perfectamente complementarias, de forma que la existencia de elementos transculturales no excluye la presencia de diferencias culturales en la expresión facial (Iglesias y cols., 1984). Así, Friesen (1972, citado por Ekman, 1973) ha observado que tanto los sujetos occidentales como los orientales muestran expresiones faciales muy similares en respuesta a películas neutras y estresantes, pero también que las respuestas expresivas de ambos grupos difieren cuando en la sala de proyección están presentes los experimentadores; en esta condición, los orientales enmascaran sus expresiones negativas ante escenas desagradables por medio de sonrisas.

Para explicar este fenómeno, Ekman y Friesen (1975) propusieron el concepto de «normas expresivas» (display rules), entendiendo por ello las normas que en cada cultura regulan la expresión social de las emociones, siendo este aspecto el menos investigado por los psicólogos hasta el momento actual (véase, p. e., Saarni, 1982). A continuación se exponen sucintamente los diferentes estudios que apoyan la hipótesis de la universalidad y el posible innatismo de las expresiones faciales de las emociones básicas.

\section{Estudios transculturales}

Los estudios llevados a cabo por Izard (1971), con sujetos occidentales coinciden con los realizados por Ekman y cols. (Ekman y Friesen, 1971), quienes incluyeron en sus trabajos individuos procedentes de culturas preletradas. En síntesis, estos autores han demostrado que los sujetos pertenecientes a catorce culturas diferentes son capaces de expresar y de reconocer las emociones supuestamente básicas de alegría, ira, miedo, sorpresa, desagrado y tristeza (Ekman y Oster, 1979/1981).

Limitándonos a los trabajos de Ekman y cols., con culturas preletradas, estos autores encontraron por una parte, que en distintas subculturas de Nueva Guinea y Borneo se reconocían de forma adecuada las expresiones faciales antes mencionadas, todas ellas simuladas por sujetos occidentales, si bien debe señalarse que los individuos pertenecientes a la subcultura Fore, de Nueva Guinea, identificaron correctamente la expresión de miedo tan sólo cuando tenía que discriminarse de las expresiones de alegría, ira y desagrado, pero no cuando debía discriminarse de la expresión de sorpresa.

Por otra parte, los autores citados no sólo demostraron que los sujetos de culturas preletradas reconocían las poses de emociones de sujetos occidentales, sino también que estos últimos eran capaces de reconocer las poses de aquéllos. Así, se observó que diferentes muestras de estudiantes norteamericanos reconocían las expresiones de alegría, ira y desagrado pertenecientes a individuos de la subcultura Fore. Los juicios volvieron a ser inadecuados con las poses de sorpresa y miedo, precisamente las mismas que los individuos Fore tenían dificultad en reconocer.

\section{Estudios con invidentes}

Distintos autores han destacado la semejanza entre la expresión emocional de sujjetos ciegos y videntes (p. e., Fulcher, 1942). Cabe destacar en especial los trabajos de Eibl-Eibesfeldt (1972, 1975), que analizó filmaciones correspondientes a niños ciegos sordomudos de diferentes edades tomadas en distintas con- 
diciones, observando que dichos sujetos presentaban los movimientos expresivos propios de la risa, sonrisa, ira, llanto, sorpresa, aceptación y rechazo. Este autor también encontró que incluso los niños ciegos, sordomudos y mutilados de ambos brazos poseen igualmente un repertorio normal de expresiones emocionales.

En esta misma línea, Ortega, Iglesias, Fernández-Dols y Corraliza (1983) han observado recientemente que las expresiones de alegría correspondientes a un grupo de niños invidentes son reconocidas adecuadamente por un grupo de jueces, y que dichas expresiones implican la actividad de los mismos músculos faciales que en los sujetos videntes. En este trabajo se plantea de nuevo que el aprendizaje visual no es una condición necesaria para el desarrollo de la expresión facial (véase también Ortega, 1982).

\section{Estudios con primates no humanos}

A lo largo de este siglo, son varios los autores que han destacado la existencia de semejanzas morfológicas entre las expresiones faciales humanas y las de otros primates no humanos (p. e., Andrew, 1963, 1972), Huber (1931), destacó que la musculatura facial indispensable para la existencia de un repertorio expresivo amplio surge en los primates a partir de una reducción de la musculatura auricular y de un incremento de la musculatura en la región media de la cara. En el hombre, por ejemplo, destaca una mayor diferenciación de los músculos en esa región, además de una disminución de su tamaño (véase también Chevalier-Skolnikoff, 1973).

Por su parte, Van Hooff (1972) ha pretendido explicar la evolución de la sonrisa y la risa humanas a partir de las expresiones «mostrar los dientes en silencio» y «boca abierta relajada» (cara de juego), que se encuentran en la mayoría de los primates no humanos y de forma más sobresaliente en los antropoides. Recientemente, Redican (1982) ha subrayado la existencia de una semejanza no sólo morfológica sino también funcional entre las expresiones faciales humanas y las propias de otros primates. El autor citado relaciona las expresiones faciales de miedo y sorpresa humanas con las expresiones «mueca» y «mueca de boca abierta», la ira con la expresión de «boca tensa», la alegría con la «boca abierta relajada» (cara de juego) y sus movimientos asociados, y la tristeza con el «puchero».

Por último, cabe mencionarse un estudio clásico de Foley sobre reconocimiento de expresiones emocionales de chimpancés por sujetos humanos, en el que se observó que un grupo de estudiantes universitarios eran capaces de juzgar correctamente las expresiones de tristeza, alegría, miedo e ira de los chimpancés. En este trabajo, tan sólo dos expresiones, calma y excitación, no se reconocieron adecuadamente, las cuales, según Chevalier-Skolnikoff, no fueron representaciones fiables de los estados atribuidos a ellas por Foley (véase Ekman, 1973).

\section{Expresión facial de emociones en lactantes}

Situándonos en esta misma tradición experimental, los estudios realizados por nuestro equipo de investigación han tenido por objetivo determinar si el lactante humano presenta en condiciones estimulares adecuadas las expresiones fa- 
ciales supuestamente universales, correspondientes a las emociones consideradas básicas e innatas por los autores antes citados. En diversos trabajos previos, la expresión facial infantil se ha estudiado mediante juicios subjetivos y también por medio de códigos observacionales. Los estudios de juicios consisten, generalmente, en solicitar a diferentes observadores que seleccionen de una lista cerrada de términos emocionales aquél que defina mejor una determinada expresión facial. A diferencia de los estudios de juicios, los estudios observacionales tienen por objetivo la medición directa de los cambios faciales que caracterizan una determinada emoción, por lo que también se denominan estudios de componentes expresivos. Los estudios de juicios, por tanto, son menos precisos que los estudios de componentes, ya que, por ejemplo, en los primeros no se tienen en cuenta los elementos configuracionales en los que se basa cada observador para emitir un juicio acerca de una determinada expresión facial (Izard y Dougherty, 1982).

A diferencia de los estudios de juicios, los códigos observacionales se componen de categorías discretas de conducta facial, las cuales pueden definirse partiendo de alguna teoría sobre la expresión emocional, de criterios funcionales o también del estudio de la base muscular de cada movimiento expresivo. Los primeros están fundamentados en teorías que presuponen la universalidad de ciertos movimientos expresivos que aparecen asociados a las emociones consideradas básicas, mientras que los segundos, también denominados etológicos o inductivos, están definidos por un número variable de categorías inferenciales, las cuales incluyen diversos movimientos expresivos que se presupone cumplen alguna función específica ante determinadas condiciones estimulares. El problema que presentan los códigos etológicos, al igual que los basados en teorías, es que son incompletos y a veces poco precisos, debido a la falta de validez de las categorías de conducta facial que los componen. Así, por ejemplo, ambos tipos de códigos incluyen categorías que reflejan acciones musculares complejas, resultantes de varias acciones faciales independientes que no tienen por qué aparecer siempre asociadas (veánse Ekman, 1982; Scherer y Ekman, 1982).

Para salvar los inconvenientes que presentan los códigos observacionales basados en teorías y los etológicos, se han desarrollado los denominados códigos anatómicos, cuyas categorías se elaboran a partir del análisis de los cambios faciales que produce la estimulación eléctrica y la contracción voluntaria de los distintos músculos faciales, esto es, de acuerdo con criterios causales. El Facial Action Coding System (FACS) de Ekman y Friesen (1978), es un ejemplo de código anatómico. El Facs se compone de 33 categorías elementales de conducta facial, denominadas unidades de acción facial, claramente diferenciadas en términos de su base muscular y de los cambios de apariencia visibles que permiten su identificación. Este código, por tanto, es potencialmente comprehensivo y el único que permite evaluar cambios evolutivos de forma objetiva y precisa (Oster, 1978).

Un segundo aspecto que debe tenerse presente en los estudios sobre expresión facial infantil son los estímulos utilizados para provocar las emociones básicas. En general, los autores han coincidido en las condiciones empleadas para producir alegría, desagrado y tristeza, pero han diferido en las condiciones que se han supuesto desencadenantes de miedo, ira y sorpresa. En estos tres últimos casos, no siempre se han aplicado estímulos adecuados. Así, el miedo se ha pretendido observar ante caídas bruscas desde una pequeña altura, ruidos intensos y también en respuesta a leves pinchazos, mientras que la ira se ha intentado provocar mediante la vacunación y la demora en la ingesta de alimentos (Emde, 
Kligman, Reich y Wade, 1978). Los pinchazos, la vacunación y las caídas bruscas son estímulos específicos de dolor, una respuesta que se define por unos movimientos expresivos muy distintos de los que se asocian con las emociones de ira o miedo. Lo mismo ocurre con el hambre producido por el retraso en la alimentación, que se caracteriza por unos componentes configuracionales expresivos distintos de los que definen la ira. Por último, los ruidos intensos originan reacciones de sobresalto o respuestas defensivas, que algún autor no considera equivalentes al miedo, la sorpresa o ninguna otra emoción (véase Iglesias, 1986).

Una vez destacados los dos aspectos que nos parecen más relevantes en el estudio de la expresión emocional infantil, pasamos a describir las principales investigaciones que hemos desarrollado hasta ahora. Brevemente, los trabajos realizados han consistido en estudiar mediante la técnica «FACS» si los lactantes de tres a nueve meses presentan expresiones faciales claramente diferenciadas ante estímulos emocionales adecuados. Las hipótesis de partida en estos trabajos se han tomado de los estudios llevados a cabo por Ekman y Friesen (1978), quienes han descrito en sujetos adultos los movimientos expresivos prototípicos de las seis emociones que estos autores consideran básicas: alegría, ira, miedo, sorpresa, desagrado y tristeza (véase la Tabla 1).

\section{TABLA I}

Prototipos fundamentales de expresión emocional según Ekman y Friesen (1978)

\begin{tabular}{|c|c|c|}
\hline Emoción & Base muscular de la expresión & Acción principal \\
\hline Alegría & $\begin{array}{l}\text { Cigomático mayor. } \\
\text { Orbicular de los párpados, por- } \\
\text { ción orbitaria. }\end{array}$ & $\begin{array}{l}\text { Retrae oblicuamente las comisu- } \\
\text { ras de los labios. } \\
\text { Eleva las mejillas }\end{array}$ \\
\hline Ira & $\begin{array}{l}\text { Superciliar; piramidal; depresor } \\
\text { superciliar. } \\
\text { Elevador del párpado superior. } \\
\text { Orbicular de los párpados, por- } \\
\text { ción parpebral. } \\
\text { Elevador propio del labio supe- } \\
\text { rior. } \\
\text { Orbicular de los labios. } \\
\text { Relajación del masetero, del } \\
\text { temporal y del pterigoideo inter- } \\
\text { no. }\end{array}$ & $\begin{array}{l}\text { Aproximan y descienden las ce- } \\
\text { jas. } \\
\text { Eleva el párpado superior. } \\
\text { Eleva el párpado inferior. } \\
\text { Eleva el labio superior. } \\
\text { Estrecha los labios. } \\
\text { Desciende la mandíbula. }\end{array}$ \\
\hline Miedo & $\begin{array}{l}\text { Frontal, porciones medial y late- } \\
\text { ral. } \\
\text { Superciliar; piramidal; depresor } \\
\text { superciliar. } \\
\text { Elevador del párpado superior. } \\
\text { Risorio. } \\
\text { Cuadrado de la barba, o relaja- } \\
\text { ción de la borla del mentón o del } \\
\text { orbicular de los labios. }\end{array}$ & $\begin{array}{l}\text { Eleva las cejas. } \\
\text { Aproxima las cejas. } \\
\text { Eleva el párpado superior. } \\
\text { Alarga las comisuras de los la- } \\
\text { bios. } \\
\text { Separa los labios. }\end{array}$ \\
\hline Sorpresa & $\begin{array}{l}\text { Frontal, porciones medial y late- } \\
\text { ral. } \\
\text { Elevador del párpado superior. }\end{array}$ & $\begin{array}{l}\text { Eleva las cejas. } \\
\text { Eleva el párpado superior. }\end{array}$ \\
\hline
\end{tabular}


TABLA I (cont.)

\begin{tabular}{|c|c|c|}
\hline Emoción & Base muscular de la expresión & Acción principal \\
\hline - & $\begin{array}{l}\text { Relajación del masetero, del } \\
\text { temporal y del pterigoideo inter- } \\
\text { no. }\end{array}$ & Desciende la mandíbula. \\
\hline \multirow[t]{2}{*}{ Desagrado } & $\begin{array}{l}\text { Elevador común del labio supe- } \\
\text { rior y del ala de la nariz. } \\
\text { Cuadrado de la barba. } \\
\text { Triangular. }\end{array}$ & $\begin{array}{l}\text { Arruga la nariz. } \\
\text { Desciende el labio inferior. } \\
\text { Desciende las comisuras de los } \\
\text { labios }\end{array}$ \\
\hline & $\begin{array}{l}\text { Relajación del masetero, del } \\
\text { temporal y del pterigoideo inter- } \\
\text { no. }\end{array}$ & Desciende la mandíbula. \\
\hline \multirow[t]{3}{*}{ Tristeza } & $\begin{array}{l}\text { Frontal, porción medial. } \\
\text { Superciliar; piramidal; depresor } \\
\text { superciliar. }\end{array}$ & $\begin{array}{l}\text { Eleva la cabeza de las cejas. } \\
\text { Aproxima las cejas. }\end{array}$ \\
\hline & Triangular & $\begin{array}{l}\text { Desciende las comisuras de los } \\
\text { labios. }\end{array}$ \\
\hline & Borla del mentón. & Eleva la barbilla. \\
\hline
\end{tabular}

Un aspecto destacable de nuestras investigaciones es la utilización de estímulos que parecen ser condiciones universales para provocar las emociones esperadas. La alegría se intentó producir durante la interacción cara a cara con la madre u otra persona familiar y, en ocasiones, recurriendo al juego «cucú-tras»; la ira, por medio de la retirada varias veces consecutivas de algún objeto de las manos o la boca del niño a la vez que se le inmovilizaban los brazos; el miedo, mediante la aproximación de un extraño; la sorpresa, provocando la aparición y desaparición repentina de alguna persona u objeto familiar al niño; el desagrado, dándole a probar zumos ácidos y, en algún caso, un chupete; y la tristeza, por último, a causa de la pasividad de la madre ante la mirada y las vocalizaciones de su hijo después de un periodo prolongado de exposición ante un extraño (Iglesias, 1986; Iglesias, Naranjo, Peláez, Becerra y Loeches, 1984).

Las respuestas faciales de los lactantes ante los estímulos indicados fueron grabadas en vídeo, para ser analizadas posteriormente cuadro a cuadro y a distintas velocidades de reproducción. Como resultado de este análisis minucioso, hemos podido observar en la conducta facial la presencia de los prototipos expresivos supuestamente universales (véase la Tabla 2). La única excepción que cabe destacar es la elevación de las mejillas y el alargamiento de las comisuras de los labios en el caso de la ira en lugar de otros cambios esperados, debido a las alteraciones respiratorias que producen las vocalizaciones que acompañan la expresión de ira infantil (para una discusión de este resultado, véase Iglesias, Loeches y Serrano, en prensa).

Debe subrayarse la clara diferenciación entre los componentes expresivos que aparecieron en cada condición estimular. Las únicas respuestas faciales que compartieron los mismos componentes expresivos en la región inferior de la cara fueron las correspondientes a las condiciones de ira y miedo. Sin embargo, la apariencia visible de la boca en ambas condiciones fue claramente distinta, debido a la presencia de la elevación de las mejillas en la supuesta expresión de ira infantil. En este caso, dicha elevación de las mejillas, producida por la contracción de la porción orbitaria del músculo orbicular de los párpados, atrae las comisuras de los labios de forma que la boca aparece con unos ángulos muy abiertos; en cambio, en el caso de la supuesta expresión de miedo, la boca se muestra 


\section{4}

TABLA II

Respuestas faciales de los lactantes ante diferentes estimulos emocionales

\begin{tabular}{|c|c|c|c|c|c|c|c|c|c|c|c|c|}
\hline \multirow[b]{3}{*}{ Condición } & \multicolumn{12}{|c|}{ Región facial } \\
\hline & \multicolumn{2}{|c|}{ Frente } & \multicolumn{3}{|c|}{ Ojos } & \multirow{2}{*}{$\begin{array}{c}\text { Nariz } \\
9\end{array}$} & \multicolumn{6}{|c|}{ Boca } \\
\hline & 1 & 2 & 4 & 5 & 6 & & 12 & 15 & 16 & 17 & 20 & $25-26-27$ \\
\hline Alegría & & & & & $\bar{X}$ & & $\mathrm{X}$ & & & & & $\mathrm{X}$ \\
\hline Ira & & & $\mathrm{X}$ & & $\mathrm{X}$ & & & & & & $\mathrm{X}$ & $\mathrm{X}$ \\
\hline Desagrado & & & & & & $\mathrm{X}$ & & $\mathrm{X}$ & $\mathrm{X}$ & $\mathrm{X}$ & & $\bar{X}$ \\
\hline Miedo & $\mathrm{X}$ & $\mathrm{X}$ & $\mathrm{X}$ & $\mathrm{X}$ & & & & & & & $\mathrm{X}$ & $\mathrm{X}$ \\
\hline Tristeza & $\mathrm{X}$ & & $\bar{X}$ & & & & & $\mathrm{X}$ & & $\mathrm{X}$ & & $\mathrm{X}$ \\
\hline Sorpresa & $\mathrm{X}$ & $\mathrm{X}$ & . & $\mathrm{X}$ & & - & & & & & & $\mathrm{X}$ \\
\hline
\end{tabular}

Componentes expresivos: 1 , elevación de la cabeza de las cejas; 2 , id. de la parte posterior de las cejas; 4 , descenso y aproximación de las cejas; 5 , elevación del párpado superior; 6, elevación de las mejillas; 9, arrugamiento de la nariz; 12, elevación de las comisuras de los labios; 15 , descenso de las comisuras de los labios; 16, descenso del labio inferior; 17, elevación de la barbilla; 20, alargamiento de las comisuras de los labios; 25-26-27, apertura de la boca. La base muscular de estos cambios faciales puede consultarse en la Tabla I.

más ovalada y con unos ángulos más agudos. Estos resultados contradicen la opinión de los autores que han sostenido que la conducta facial infantil no está suficientemente diferenciada. Como se verá a continuación, esta diferenciación también se encuentra en la capacidad del lactante para discriminar entre distintas expresiones emocionales.

\section{Reconocimiento de expresiones faciales en lactantes}

Los estudios sobre reconocimiento de expresiones faciales de emociones en lactantes no se consideran relevantes hasta que se advierte la existencia de un conocimiento social temprano, manifestado por la atracción visual del neonato por la cara humana frente a otros estímulos visuales (Fantz, 1963). Sin embargo, hasta ahora los psicólogos evolutivos han afrontado el estudio de la conducta infantil como un modelo para contrastar diversas hipótesis acerca del desarrollo de los procesos cognitivos, dejando de lado el estudio de la emoción (Wolff y Ferber, 1979). Quizá por esta razón los autores que realizan las primeras investigaciones sistemáticas sobre discriminación y reconocimiento de expresiones faciales de emociones en lactantes no relacionan sus resultados con los estudios sobre expresión emocional infantil, salvo alguna excepción (p. e., Oster, 1981).

Si partimos de los presupuestos psicobiológicos que asumen las distintas teorías neodarwinianas actuales de la emoción (p. e. Izard, 1977), la evolución de señales visuales específicas para cada emoción habría ocurrido a la par que una presión selectiva destinada a mejorar la capacidad de respuesta frente a esas señales (véanse también Hinde, 1974/1977; Lewis y Gower, 1980; Sackett, 1963; Smith, 1977/1982). En consecuencia, el lactante debería ser capaz de discriminar y reconocer las expresiones faciales supuestamente universales en la cara de una persona adulta.

Centrándonos en los escasos trabajos existentes, debemos destacar en pri- 
mer lugar que el reconocimiento de expresiones faciales en la infancia se ha estudiado por medio del análisis del comportamiento motor y de la atención visual del lactante ante distintas expresiones emocionales. Hasta ese momento, la fijación visual es la variable dependiente que parece ser más sensible ante las diferentes expresiones faciales de emociones. Por ello, el paradigma de investigación más utilizado por los autores es el de habituación visual (Oster, 1981).

El paradigma de habituación conlleva una primera fase de familiarización y una segunda posterior de discriminación. En la fase de familiarización, se le presenta al lactante una determinada expresión facial durante varios ensayos consecutivos hasta que apenas se fija en ella. A continuación, en la fase de discriminación, se le presenta sucesivamente una expresión facial nueva y la familiar. Si un niño, tras la fase de familiarización con una expresión (p. e., alegría) mira más a una segunda distinta de la anterior (p. e., miedo), se asume entonces que las ha discriminado. Además, si la fijación visual del lactante decae al presentarse nuevamente la expresión familiar (alegría), se infiere que la ha reconocido (Nelson, 1987; Young-Browne, Rosenfeld y Horowitz, 1977).

El segundo aspecto que debe tenerse en cuenta en un estudio sobre discriminación de expresiones faciales en la infancia son los estímulos presentados. Los investigadores han expuesto a los lactantes ante dibujos esquemáticos, diapositivas, películas y también ante modelos naturales simulando diferentes emociones. Desde nuestro punto de vista, ni los dibujos, ni las películas, ni tampoco los modelos naturales son estímulos adecuados si lo que pretendemos es conocer la sensibilidad del lactante ante diferentes expresiones faciales.

Por una parte, los dibujos tan sólo incluyen algunos de los componentes expresivos que definen una determinada emoción y constituyen, por lo general, representaciones poco fiables de la cara humana. Por otra parte, los estudios llevados a cabo con películas y sobre todo con modelos naturales confunden la estimulación facial con otros estímulos que por sí mismo producen cambios en la fijación visual del lactante (p. e., movimientos de cabeza o vocalizaciones). Además, en este tipo de estudios es preciso evaluar la fiabilidad y consistencia de las poses, ya que los modelos naturales reproducen por lo general poses muy ambiguas o también poses que contienen componentes expresivos de dos emociones distintas. A causa de las objeciones señaladas, las diapositivas de expresiones faciales son el tipo de estímulo más idóneo para nuestro propósito. Sin embargo, llama la atención que, en general los autores no hayan reparado en la importancia de validar mediante criterios objetivos las diapositivas utilizadas en sus investigaciones (Iglesias, 1986).

Teniendo en cuenta lo anterior, los trabajos que hemos realizado en esta línea han consistido en estudiar mediante un paradigma de habituación visual si los lactantes de tres a nueve meses son capaces de discriminar entre diapositivas en color de expresiones faciales de alegría, ira, miedo, sorpresa, desagrado y tristeza. Dichas expresiones pertenecieron a una mujer joven y se seleccionaron entre un total de 72 poses por medio de la técnica «FACS» que permite identificar la presencia de los componentes expresivos que, según Ekman y Friesen (1978), son universales y característicos de cada emoción. Las expresiones seleccionadas se proyectaron en diferentes sesiones, balanceando su orden de presentación entre los sujetos estudiados. En cada sesión, el lactante tenía la oportunidad de ver dos expresiones, una familiar y otra nueva, hasta completar el conjunto de condiciones resultantes de combinar las seis expresiones por pares.

Los resultados obtenidos pusieron de manifiesto que todos los sujetos dis- 
criminaron las diferentes expresiones presentadas, ya que siempre miraron más a la expresión nueva ( $\bar{x}=9,3$ segs), que a la habituada ( $\bar{x}=2,9$ segs.) Dado que el mismo modelo femenino posó las seis emociones, los estímulos presentados al lactante difirieron tan sólo en los componentes expresivos que son características de cada emoción. De aquí se puede deducir que los cambios en la fijación visual del lactante ante cada expresión nueva pudieron deberse a dichos componentes expresivos y no al contorno pelo-cara $\mathrm{u}$ otros indicios externos (Iglesias, 1986; Iglesias, Naranjo, Peláez y Ortega, 1985).

Por otra parte, los lactantes no sólo demostraron unos tiempos de fijación superiores en cada expresión nueva con respecto a la familiar o habituada sino que también presentaron distintas preferencias visuales según la expresión facial nueva de que se trate. Así, las expresiones denominadas positivas (p. e., alegría y sorpresa) fueron siempre más atendidas que las negativas (p. e., miedo y desagrado) como puede apreciarse en la Figura 2.

En un segundo estudio decidimos presentar dichos modelos femeninos en

FIGURA 2

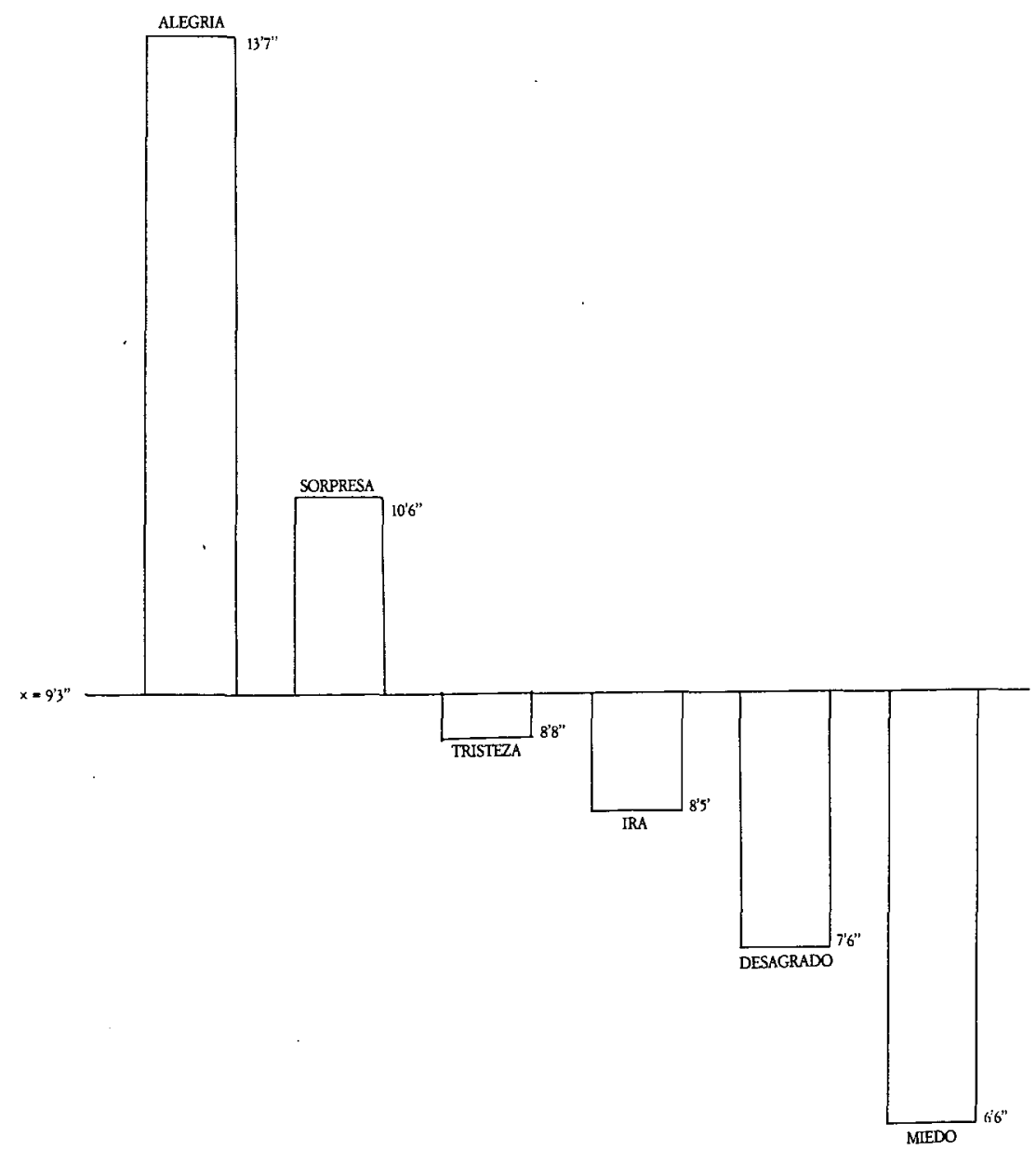

Diagrama de barras ilustrando los tiempos medios de fijación de los lactantes en cada expresión facial nueva durante la fase de discriminación visual. Las diferencias existentes entre las expresiones quedan reflejadas por las desiraciones entre los distintos tiempos de fijación con respecto a la media global. 
la fase de familiarización y en la de prueba, para demostrar si el lactante es capaz de generalizar la discriminación de expresiones faciales a modelos que no ha visto con anterioridad. En este caso, las expresiones presentadas fueron las prototípicos de ira, miedo y sorpresa, las cuales fueron validadas siguiendo el mismo procedimiento que en el caso anterior. En general, se encontró que los niños de cuatro a seis meses discriminaron y generalizaron el reconocimiento de expresiones faciales de ira, miedo y sorpresa posadas por varios modelos femeninos (Iglesias, Loeches y Serrano, 1988; Serrano, 1986). De acuerdo con Caron, Caron y Myers (1982), puede decirse que los lactantes percibieron las emociones basándose en un esquema o patrón conceptual, esto es, que reconocieron la invarianza de los prototipos expresivos que representaban una misma emoción abstrayendo sus características comunes, y que mediante dicha abstracción discriminaron las distintas expresiones emocionales.

El problema que intentamos resolver en dos estudios posteriores era determinar si la respuesta visual del lactante estaba basada realmente en los elementos expresivos que caracterizan las distintas emociones. Para ello, analizamos mediante la técnica «FACS» las respuestas faciales de niños de cuatro a seis meses de edad ante expresiones de alegría, tristeza ira y miedo. Encontramos que los lactantes tienden a reproducir los mismos cambios expresivos que observan en la pose del adulto, aunque sin llegar a imitar la configuración total, lo que apoya indirectamente la hipótesis inicial de que la discriminación visual de expresiones faciales en lactantes se basa en los componentes expresivos que son específicos de cada emoción (Iglesias y cols., 1988; Sánchez, 1986; Serrano, 1987).

Un dato concreto que debe destacarse de los resultados de nuestros trabajos es que los lactantes muestran diferentes tiempos de fijación visual ante cada ex. presión facial en la fase de discriminación o de prueba, de forma que las expresiones consideradas positivas, como la alegría, parecen ser «preferidas» ante otras supuestamente negativas, como el miedo o el desagrado (véase la Figura 2). Este dato puede ser un indicativo de que los lactantes no sólo discriminan visualmente las distintas expresiones faciales, sino que también reconocen su distinto valor funcional. Lo anterior es congruente con la observación de que los niños, desde los cinco meses, lloran ante caras de ira y sonríen ante caras de alegría (sobre este punto, véase Iglesias, 1986; Serrano, 1986), debiendo determinarse en futuros estudios si los lactantes reconocen realmente la señal de amenaza que conllevan las expresiones de ira, la indicación de peligro que se asocia a la expresión de miedo, la necesidad de ayuda que implica una expresión de tristeza o la buena intención y el carácter reforzante de una expresión de alegría.

Por último, como extensión de los trabajos anteriores sobre expresión facial y reconocimiento de emociones en lactantes normales, hemos realizado recientemente investigaciones con lactantes con síndrome de Down (trisomía del par de autosomas 21) con edades comprendidas entre tres y once meses. Siguiendo la metodología empleada en nuestros estudios con niños normales, encontramos que los niños afectados por el síndrome de Down mostraron también expresiones faciales claramente diferenciadas de alegría, ira, miedo, sorpresa, desagrado y tristeza y que, además, la musculatura facial implicada en la producción de dichas expresiones emocionales es la misma que subyace a las correspondientes de los niños normales. Asimismo, los lactantes con sindrome de Down mayores de seis meses fueron capaces de discriminar entre expresiones tales como alegría, ira y miedo, mostrando las mismas preferencias visuales que los niños normales (Loeches, 1988). 
Naturaleza de las expresiones emocionales

Aún se desconoce si otras emociones supuestamente básicas para algunos autores (p. e., interés y desprecio) están definidas por una expresión facial universal (Ekman y Oster, 1979/1981). Creemos que ello se debe a que todavía se ignoran los mecanismos evolutivos que dan lugar a la génesis de las expresiones faciales. Con otras palabras, no existe ninguna teoría actual que explique por qué determinados movimientos expresivos se asocian diferencialmente a cada emoción. Mientras que algunos autores presuponen que las expresiones faciales de las emociones básicas son innatas (p. e., Izard, 1971), otros consideran que emergen en el curso del desarrollo de forma gradual a partir de respuestas tales como la succión y el llanto (p. e., Peiper, 1963). Considerando ambas posiblidades, el propio Darwin (1872/1984) propuso los tres principios siguientes para explicar el origen de las expresiones faciales: 1) principio de los hábitos útiles asociados, 2) principio de la antítesis y 3) principio de la acción directa del sistema nervioso (véanse Bühler, 1933/1980; Montgomery, 1985).

Dado que los movimientos expresivos son consecuencia de la contracción y relajación de los músculos faciales, es obvio que el primer paso que ha de darse para comprender su naturaleza consiste en examinar con detalle la anatomía funcional de la cara de los primates. Lo primero que se advierte es que los músculos faciales se encuentran situados en torno a los orificios craneofaciales; su contracción o relajación produce los distintos grados de apertura u oclusión de estos orificios y el movimiento de los apéndices de la cabeza (cejas, párpados, orejas y nariz). Así los músculos faciales participan directamente en procesos tan variados como son la visión, audición, olfacción, respiración, succión, deglución, masticación y fonación, a la vez que contribuyen a proteger los órganos sensoriales frente a determinados cambios ambientales (Gasser, 1967; Huber, 1931). En consecuencia, tal y como indicaba Darwin, es muy posible que ciertas expresiones faciales de las emociones se hayan originado a partir de acciones que inicialmente cumplan una función meramente instrumental.

Un segundo paso para determinar la naturaleza de las expresiones faciales consiste precisamente en explicar cómo las diferentes acciones de los músculos faciales se han asociado para formar configuraciones expresivas, las cuales habrían ocurrido cada vez con una mayor frecuencia hasta convertirse en señales estereotipadas que intervienen en la regulación de las interacciones sociales intraespecíficas (Andrew, 1963, 1972). A este proceso evolutivo se le conoce con el nombre de ritualización (Huxley, 1966).

En la actualidad, se dispone de hipótesis muy parciales acerca de la naturaleza de algunos de los movimientos expresivos que participan en las distintas emociones consideradas básicas. La sorpresa, por ejemplo, está caracterizada por la contracción del músculo frontal, porciones medial y lateral, que produce la elevación y el arqueo de las cejas. Esta acción da lugar a un aumento de la parte superior del campo visual, y en consecuencia, favorece la percepción de estímulos externos. Prueba de ello es la realización voluntaria de este movimiento bajo los efectos del sueño y la embriaguez, contrarrestando así la relajación del músculo elevador del párpado superior que es notoria en dichos estados. Por su parte, Darwin (1872/1984) subrayó que la contracción del músculo frontal contribuye a levantar el párpado superior con mayor rapidez, liberando el globo ocular con el objeto de facilitar la búsqueda visual.

Otro movimiento expresivo de interés es la elevación de las cejas a la vez 
que su fruncimiento en las expresiones de miedo, interviniendo en este caso, además del músculo frontal, la acción sinérgica de los músculos superciliar, piramidal y depresor superciliar. Ekman (1979), señala que este movimiento puede que resulte de una reacción ante un estímulo novedoso y de la percepción posterior de una amenaza, habiéndose especializado como una señal que indica la anticipación de un peligro.

Algunos autores han insistido en la importancia del hábitat para analizar la génesis de las expresiones faciales (Andrew, 1963; Redican, 1975). Así, los prosimios por ser especies nocturnas, carecen de movimientos expresivos, a no ser acciones relacionadas con la olfacción y la audición (Chevalier-Skolnikoff, 1973). En cambio, los monos del Viejo Mundo, que viven en espacios terrestres abiertos, muestran un repertorio facial más rico y una actividad muscular mayor alrededor de la boca que los del Nuevo Mundo, que habitan en los árboles (Andrew, 1972).

También se ha señalado por último, la existencia de repertorios amplios de señales visuales en aquellas especies que viven en grupos sociales grandes, como sucede con los papiones (Naranjo, 1985; Van Hoof, 1972). Por todo ello, es razonable esperar que la naturaleza de las expresiones faciales pueda ser explicada mejor a partir de nuevos estudios comparativos entre primates humanos y no humanos, no sólo morfológicos, sino también funcionales. En el momento actual, este es uno de los problemas teóricos más importantes que debe acometerse para poder avanzar en este campo.

\section{Referencias}

AlLPORT, F. H. (1967). Social psychology, Nueva York, Johnson Reprint Corporation, Reimpresión del original publicado en 1924 .

ANDREW, R. (1963). The origin and evolution of the calls and facial expressions of the primates, Behaviour, 20, 1-109.

- (1972). The information potentially available in mammal dysplays. En R. A. Hinde (Ed.): Nonverbal communication. Cambridge: Camgridge University Press.

BLANCHARD, D. C. y BlanCHARD, R. J. (1988). Ethoexperimental approaches to the biology of emotion. Annual Review of Psychology, 39, 43-68.

Blankstein, D. R., PlinNer, P. y POLIVY, J. (eds.). (1980). Advances in the study of communication and affect (vol. 6): Assesment and modification of emotional bebavior, Nueva York, Plenum Press.

BLuRTON.JONES, N. G. (1971). Criteria for use in describing facial expressions in children, Human biology, 41, 365-413.

BUHLER, K. (1933). Ausdruckstheorie, Das sytem an der geschicbte aufgezeight, Stuttgart, Gustav Fischer Verlag. Traducción castellana: Teoria de la expresión, Madrid, Alianza, 1980.

CARON, R. F.; CARON, A. J. y MYERS, R. S. (1982): Abstraction of invariant face expressions in infancy, Child Development, 53, 1008-1015.

CHEvalier.SKOLNIKOFF, S. (1973). Facial expression of emotion in nonhuman primates. En P. Ekman (ed.): Daruin and facial expression, Nueva York: Academic Press.

CLARK, M. S. y FISKE, S. T. (eds.) (1982). Affect and cognition: the seventeeth annual carnegie symposium on cogniton, Hillsdale, N. J.: LEA.

DARWIN, C. (1972). The expression of emotions in man and animals, Londres, J. Murray. Traducción castellana: La expresión de las emociones en los animales y en el bombre, Madrid, Alianza, 1984.

- (1877). A biographical sketch of an infant. Mind, 2, 285-294. Traducción castellana: Ensayo sobre el instinto y apunte biográfico de un niño, Madrid, Tecnos, 1983.

EIBL-EIBESFELDT, I. (1972). The cross-cultural documentation of social behavior. En R. Chauvin (Ed.). Modeles animaux du comportement bumain, Paris,CNRS.

- (1975). Concepts of ethology and their significance in the study of human behavior. En H.W. Stevenson, E. H. Hess, y H. L. Rheingold (eds.): Early Bebavior. Huntington, N. Y.: Krieger.

Ekman, P. (1973). Daruin and facial expresssion. Nueva York. Academic Press.

- (1977). Biological and Cultural contributions to body and facial movement. En I.Blacking (ed.): The antbropology of the body. Londres, Academic Press.

- (1979). About brows. Emotional and conversational signals. En M. von Cranach, K. Foppa, W. Lepenies y D. Ploog (eds.); Human etbology. Cambridge, Cambridge University Press. 
- (1982). Emotion in the human face (segunda ed. rev.) Cambridge: Cambridge University Press.

ExMAN, P. y FriesEN, W. V. (1971). Constants across cultures in the face and emotion. Journal of Personality and Social Psycbology, 17, 124-129.

- (1975). Unmasking the face. Englewood Cliffs, N. Y.: Prentice-Hall.

- (1978). The facial action coding system (FACS). Palo Alto, California. Consulting Psychologists Press.

Ekman, P. y Oster, H. (1979). Facial expresssions of emotion. Annual Review of Psychology, 30, 527.554. Traducción castellana en Estudios de Psicologia, 1981, 7, 116-144.

Emde, R. N.; Krigman, D. H.; ReICH, J. H. y WadE, T. D. (1878). Emotional expression in infancy: I, Initial estudies of social signaling and an emergent model. En M. Lewis y L. A. Rosenblum (eds.); The development of affect, Nueva York, Plenum Press.

FANTZ, R. L. (1963). Patterns vision in newborn infants. Science, 140, 296-297.

Fernandez-Dols, J. M.; Iglesias, J. y MalLO, M. J. Comportamiento no verbal y emoción. En J. L. Pinillos y J. Mayor (Eds.). Tratado de Psicologia (vol. 3). Madrid, Alhambra. En Prensa.

Fox, N. A. y Davidson, R. J. (Eds.) (1984). The psychology of affective development. Hillsdale, N. J. LEA.

FrIJDA, N. H. (1986). The emotions. Cambridge, Cambridge University Press.

FULCHER, J. S. (1942). «Voluntary» facial expression in blind and seeing children, Archives of Psycho$\log y, 272,1-49$.

GASSER, R. F. (1967). The development of the facial muscles in man, American Joumal of Anatomy, $120,357.375$.

Goodenough, F. L. (1931). The expression of emotions in infancy. Child Development, 2, 96-101.

HEBB, D. O. (1946). Emotions in man and animals: an analysis of intrusive process of recognition. Psychological Review, 53, 88-106.

HINDE, R. A. (1974). Biological basis of social buman behavior. Nueva York. McGraw-Hill. Traducción castellana: Bases biológicas de la conducta social humana, México, Siglo XXI, 1977.

HUBER, E. (1931). Evolution of facial musculature and facial expression. Baltimore, Johns Hopkins Press.

HUXLEY, J. (1966). Introduction to «A discussion on ritualization of behaviour in animals and man». Philosophical Transactions of the Royal Society of London. Series B, 251 (772), 249.271.

IGLESIAS, J. (1986). Expresión facial y reconocimiento de emociones en la infancia. Tesis doctoral no publicada, Universidad Autónoma de Madrid.

Iglésias, J.; LOECHES, A. y SERRANO, J. M. (1988). Infant ability to discriminate, imitate and recognize emotional faces. Comunicación presentada en el Third European Symposium on Facial Expression Measurement and Meaning. Tagernsee, Alemania Federal, 14-18 de marzo.

- Facial expression of basic emotions in infancy. Psychophysiology, en prensa.

Iglesias, J.; Naranjo, J. M.; Pelaez, F.; Becerra, A. y Loeches, A. (1984). Les expressions faciales qui précèdent le sourire et le pleur chez le nourrison de neuf à vingt semaines. En $A$. de Haro y X. Espadaler (eds.). Processus d'acquisition précoce. Les communications. Bellaterra, Universidad Autónoma de Barcelona.

Iglesias, J.; Naranjo, J. M.; Pelaez, F. y Ortega, J. E. (1985). Perception of emotions in infants from an ethological perspective. American Journal of Primatology, 8, 344.

Igleias, J.; Naranjo, J. M.; Picazo, C. y Ortega, J. E. (1984). La cara y la emoción. Datos para una réplica. Estudios de Psicología, 18, 101-111.

IsAaCSON, R. L. (1982). The limbic system, Nueva York, Plenum Press.

IZARD, C. E. (1971). The face of emotion. Nueva York. Appleton.

- (1977). Human emotions, Nueva York. Appleton.

- (1979). The maximally discriminative facial movement coding system (MAX), Neward, Instructional Resources Center, University of Delaware.

- (1982). Measuring emotions in infants and cbildren (vol. 1). Cambridge, Cambridge University Press.

IZARD, C. E.y DOUGHERTY, L. M. (1982). Two complementary systems for measuring facial expressions in infants and children. En C. E. Izard (ed.). Measuring emotions in infants and children Vol. 1, Cambridge, Cambridge University Press.

KleinginNa, P. R. y KLeInGINNA, A. M. (1981). A categorized list or emotion definitions with suggestions for a consensual definition, Motivation and Emotion, 5, 345-379.

KuPFERMANN, I. (1985). Hypotalamus and limbic system. I: Peptidergic neurons, homeostasis, and emotional behavior. En E. R. Kandel y J. H. Schwartz (eds.). Principles of neural science (2." ed.). Nueva York, Elsevier.

LANDIS, C. (1929). The interpretation of facial expression in emotion. Journal of General Psychology, 2, 59-72.

LeVentHaL, H. y TomaRken, A. J. (1986). Emotion: Today's problem. Annual Review of Psychology, $37,565-610$.

LevI, L. (ed.) (1975). Emotions: Their parameters and measurement. Nueva York: Raven Press Publishers.

LEWIS, A. B. y GoWER, D. M. (1980). Biology of communication, Londres, Blackie.

LOECHES, A. (1988). Discriminación y expresión de emociones en lactantes con síndrome de Down. Tesis doctoral no publicada, Universidad Autónoma de Madrid.

LUTZ, C. y WHITE, G. M. (1986). The antropology of emotions. Annual Review of Antropology, 15, 405-436. 
MCGrew, W. C. (1972). An ethological study of children's behavior. Nueva York, Academic Press.

MONTGOMERY, W. (1985). Charles Darwin's thought on expressive mechanisms in evolution. En G. Ziving (Ed.). The development of expressive behavior: Biology-environment interactions, Nueva York, Academic Press.

NARANjo, J. M. (1985). Expresión facial y organización social en una colonia de babuinos cautivos (Papio bamadryas, Papio cinocephalus y sus bibridos). Tesis doctoral no publicada, Universidad Autónoma de Madrid.

NELSON, C. (1987). The recognition of facial expressions in the first two years of life: Mechanism of development. Child Development, 58, 889-909.

ÖHmAn, A. (1987). Psychophysiology of emotions. An Evolutionary-cognitive perspective. En P. K. Ackles, J. R. Jennings y M. G. H. Coles (eds.). Advances in Psychophysiology (vol. 2).Greenwich Conneticut, Jai Press.

OOMURA, I. (ed.) (1986). Emotions. Neuronal and chemical control. Basel: Carger.

ORTEGA, J. E. (1982). La expresión facial en ciegos congénitos. Tesis doctoral no publicada, Universidad Autónoma de Madrid.

Ortega, J. E.; Iglesias, J; Fernandez, Dols, J. M. y Corraliza, J. A. (1983). La expresión facial en ciegos congénitos. Infancia y Aprendizaje, 21, 83-96.

OSTER, H, (1978). Facial expression and affect development. En M. Lewis y L. A. Rosenblum (eds.): The development of affect, Nueva York, Plenum Press.

- (1981). «Recognition» of emotional expression in infancy? En M. E. Lamb y C. R. Sherrod (eds.). Infant social cognition: empirical and theoretical considerations. Hillsdale, N. J.: LEA

PANKSEPP, J. (1982). Toward a general psychobiological theory of emotions. The Behavioral and Brain Sciences, 5, 407-422.

PEIPER, A. (1963). Cerebral function in infancy and chilhood. Nueva York, Consultants Bureau.

PLUTCHIK, R. (1980). Emotion: a psychoevolutionary synthesis. Nueva York, Harper and Row.

PLUTCHIK, R. y KELLERMAN, H. (eds.) (1980). Emotion: theory, research and experiences (vol. 1). Theories of emotion. Londres, Academic Press.

REDICAN, W. K. (1975). Facial expressions in nonhuman primates. En L. A. Rosemblum (ed.). Primate behavior: Development in field and laboratory research (vol. 4), Nueva York, Academic Press.

- (1982). An evolutionary perspective on human facial displays. En P. Ekman (ed.): Emotion in the buman face (segunda ed. rev.). Cambridge, Cambridge University Press.

RINN, W. E. (1984). The neuropsychology of facial expression: a review of the neurological and psychological mechanisms for producing facial expressions. Psychological Bulletin, 95, 52-77.

SAARNI, C. (1982). Social and affective functions of nonverbal behavior: Development concerns. En R. J. Feldman (ed.). Development of nomerbal bebavior in children. Nueva York: SpringerVerlag.

SACKETT, G. P. (1963).A neural mechanism underlying unlearned, critical period and developmental aspects of visually controlled behavior, Psychological Review, 70, 40-50.

SANCHEZ, A. (1986). Imitación de expresiones faciales en la infancia. Memoria de licenciatura no publicada, Universidad Autónoma de Madrid.

SCHEFF, T. J. (1983). Toward integration in the social psychology of emotions, Annual Review of Sociology, 9, 333-354.

SCHERER, K. R. y EKMAN, P. (eds.) (1982). Handbook of metbods in nonverbal communication, Cambridge, Cambridge University Press.

- (1984). Approaches to emotion, Hillsdale, N. J., LEA.

SCHLosberg, H. (1954). Three dimensiones of emotion. Psychological Review, 61, 81-88.

SERRANO, J. M. (1987). Fijación visual y conducta facial de lactantes humanos ante expresiones emocionales. Memoria de licenciatura no publicada, Universidad Autónoma de Madrid.

- (1986). Abstracción de características invariantes de expresiones faciales de ira, miedo y sorpresa en niños de 4 a 6 meses de edad. Memoria de licenciatura no publicada, Universidad Autónoma de Madrid.

SHAVER, P. (ed.) (1984). Emotions, relationships and health. Review of Personality and Social Psycho$\log y, 7,265-284$.

SHERMAN, M. (1927). The differentiaton of emotional responses in infants I. Judgements of emotional responses from motion picture views and from actual observation, Journal of Comparative Psychology, 7, 265-284

SmiTh, O. A. y DeVITo, J. L. (1984) Central neural integration for the control of autonomic responses associated with emotion. Annual Review of Neuroscience, 7, 43-65.

SMITH, W. J. (1977). The behavior of communicating. An ethological, approach. Cambridge, Harvard University Press. Traducción castellana: Etología de la comunicación, México, Fondo de Cultura Económica, 1982.

TomkINS, S. S. (1962). Affect, imagery, consciousness (Vol. 1): The positive affects, Nueva York, Springer-Verlag.

- (1963). Affects, imagery, consciousness (vol. 2). The negative affects, Nueva York, Springer-Verlag.

- (1982). Affect theory. En P. Ekman (ed.). Emotion in the human face (2." ed. rev.). Cambridge. Cambridge University Press.

TREVARTHEN, C. (1985). Facial expressions of emótion in mother-infant interaction, Human Neurobiology, 4, (1), 21-32. 
VALLE-INCLAN, F. (1983). La cara y la emoción. Límites de la universalidad. Estudios de Psicología, $16,107-115$.

VAN.Hoof, J. A. R. A. M. (1972). A comparative approach to the phylogeny of laughter and smiling. En R. A. Hinde (ed.). Nonverbal communication, Cambridge University Press.

WEINER, B. (1986). An attributional theory of motivation and emotion. Nueva York, Springer-Verlag.

WOLFF, P. H. y FERBER, R. (1979). The development of behavior in human infants, premature and newborns. Annual Review of Neuroscience, 2, 291-307.

WoODWORTH, R. S. (1938). Experimental Psychology. Nueva York, Henry Holt.

Young-Browne, G.; Rosenfeld, H. M. y HorowITZ, F. D. (1977). Infant discrimination of facial expressions. Cbild Development, 48, 555-562.

\section{Extended summary}

This paper reviews the present state of knowledge on expression and recognition of emotions in infancy, focusing on the experimental studies carried out by our group during the last five years. The theoretical starting point of these studies is that the emotions which are considered to be basic are discrete states of the organism, phylogenetically shaped, because of their adaptative value to individuals under specific environmental needs. Such emotional states, according to data from Physiological Psychology and Neuropsychology, are controled by neural nuclei in the hypothalamus and limbic system. Based upon these theoretical grounds and considering the empirical evidence that supports the hypothesis of the universality of facial expression, we have carried out a number of investigations in order to study if human infants are able to express and recognize the emotions considered to be basic by some authors.

The greatest part of research carried out to date has focused on the description of morphological changes registered in the infants facial behaviour when faced with different emotional stimuli, often applied by their own mothers. This description has been possible by using the observational code Facial Action Coding System, which enables the identification of the different facial changes in terms of their muscular basis. As a result from these studies, we have found six prototypes of infant facial behaviour, very similar to the facial expressions of emotion described in adult and blind subjects and which are supposed to be universals.

These studies on facial expression were followed up by a group of investigations on the discrimination of facial expressions, categorical perception, and imitation of emotional gestures. By using a visual habituation-recovery paradigm, the variables studied in these new studies were visual fixation time and facial changes, both appearing as responses to facial emotional expressions posed by an adult woman. In general, we can conclude that three month-old infants are able to discriminate emotions considered to be basic by some authors when they are posed by unknown people. They also try to reproduce the same expressive movements shown by the adult. These results have also been obtained in studies on infants with Down's syndrome.

At present we try to determine when is it that infants understand the affective meaning of the different facial expressions. We are also interested in studying when and in which was voluntary control of emotional expressions appears, taking in to account that the first step in the instrumental use of smiling and crying, which can be observed at the age of three months.

A final problem that we want to point out, and which remains insolved, is the origin of facial expressions. Although it is known that the major factors 


\section{3}

which explain the origin of expressive movements are the novelty of the stimuli, the respiratory changes associated with vocalizations, and the habitat in order to progress in our present knowledge of this issue, comparative studies on facial expression of human an non human primates in similar social contexts are needed.

With these future investigations we shall have new cues for the study of human facial expression and we shall be able to extend, from a evolutionary perspective, the study of emotional development in infancy. This will help to improve our understanding of the nature and function of the different skills which make up the behavioural repertoire of the infant as well as our understanding of the nature of their first social interactions. 